\section{Über die Natur der Siliciumdioxidmodifikation Silica-X}

On the Nature of the Silica Modification "silica-X"

Klaus Beneke und Gerhard Lagaly*

Institut für anorganische Chemie der Universität Kiel,

Olshausenstraße 40/60, D-2300 Kiel

Z. Naturforsch. 34b, 648-649 (1979); eingegangen am 26. Januar 1979

Silica, Silicic Acids, Intercalation, Potassium Silicate, Silica Synthesis

Silica-X described in 1964 as a new $\mathrm{SiO}_{2}$ modification is the dehydrated form of the crystalline silicic acid $\mathrm{H}_{2} \mathrm{Si}_{8} \mathrm{O}_{17} \cdot x \mathrm{H}_{2} \mathrm{O}$ which is obtained by cation exchange from the potassium silicate $\mathrm{K}_{2} \mathrm{Si}_{8} \mathrm{O}_{17} \cdot x \mathrm{H}_{2} \mathrm{O}$.

1964 wurde von A. Heydemann [1] eine neue Siliciumdioxidmodifikation ,,Silica- $\mathrm{X}^{\text {" }}$ beschrieben Sie bildet sich unter hydrothermalen Bedingungen bei etwa $200{ }^{\circ} \mathrm{C}$ in kaliumhydroxidhaltiger Lösung (meist 2-proz. Lösungen) an Kieselgel. Das Auftreten von Silica-X als Zwischenstufe bei der Quarzbildung bei relativ niederen Temperaturen $\left(\leqslant 200^{\circ} \mathrm{C}\right)$ wurde mehrfach bestätigt $[2,3]$, doch blieb die Natur dieser Siliciumdioxidmodifikation rätselhaft. Von den anderen Siliciumdioxidmodifikationen unterscheidet sich Silica-X durch den hohen maximalen d-Wert von $1,80 \mathrm{~nm}$, die bei verschiedenen Proben stark schwankende Breite und Intensität der Röntgenreflexe und eine auffallende Verschiebung des Reflexes $d=1,80 \mathrm{~nm}$ beim Erhitzen (z.B. auf $1,73 \mathrm{~nm}$ bei $300^{\circ} \mathrm{C}[2]$ ).

Nach unseren Untersuchungen ist Silica-X die teilweise entwässerte Form einer kristallinen Kieselsäure. Unter Normaldruck bildet sich bei $100-150^{\circ} \mathrm{C}$ in kaliumhydroxidhaltigen Lösungen in einem weiten Konzentrationsbereich aus Kieselgel (typischer Ansatz: $1 \mathrm{~mol} \mathrm{KOH}, 3 \mathrm{~mol} \mathrm{SiO}_{2}, 40 \mathrm{~mol} \mathrm{H}_{2} \mathrm{O}$ ) ein quellungsfähiges Kaliumsilicat $\mathrm{K}_{2} \mathrm{Si}_{8} \mathrm{O}_{17} \cdot x \mathrm{H}_{2} \mathrm{O}$ [4], das bereits bei intensivem Waschen mit Wasser die Kaliumionen gegen Protonen austauscht und in eine kristalline Kieselsäure $\mathrm{H}_{2} \mathrm{Si}_{8} \mathrm{O}_{17} \cdot x \mathrm{H}_{2} \mathrm{O}$ übergeht. $(x \approx 0,5$ nach Gefriertrocknung [5]). Die Schichtstruktur der Kieselsäure läßt sich durch die Fähigkeit zur Einlagerung polarer Neutralmoleküle nachweisen [5]. Die dabei beobachteten Veränderungen des Röntgendiagramms zeigen, daß der maximale d-Wert der reinen Kieselsäure den Schichtabstand angibt. Dieser ist bei der reinen, voll hydratisierten Kieselsäure bei Zimmertemperatur 2,21 nm. Durch Verlust des Zwischenschichtwassers erniedrigt er sich beim Erhitzen auf höhere Temperaturen oder mit abnehmendem relativem Dampfdruck (Tab. I).

* Sonderdruckanforderungen an Prof. Dr. G. Lagaly. 0340-5087/79/0400-0648/\$01.00/0
Tab. I. Schichtabstände der kristallinen Kieselsäure $\mathrm{H}_{2} \mathrm{Si}_{8} \mathrm{O}_{17} \cdot x \mathrm{H}_{2} \mathrm{O}$ und des Kaliumsilicates $\mathrm{K}_{2} \mathrm{Si}_{8} \mathrm{O}_{17} \cdot x \mathrm{H}_{2} \mathrm{O}$ unter verschiedenen Bedingungen.

\begin{tabular}{ll}
\hline Probe & Schichtabstand [nm] \\
\hline Kieselsäure & \\
feucht & 2,21 \\
lufttrocken & 1,96 \\
über $\mathrm{P}_{4} \mathrm{O}_{10}$ & 1,80 \\
$20-40 \%$ Feuchtigkeit & 1,80 \\
nach Erhitzen auf $200^{\circ} \mathrm{C}$ & $1,73-1,78^{*}$ \\
nach Erhitzen auf $800^{\circ} \mathrm{C}$ & $1,62,1,68^{*}$ \\
nach Einlagerung von: & \\
$\quad$ Dimethylsulfoxid & 2,36 \\
$\quad$ Dimethylformamid & 2,21 \\
$\quad$ Glycol & 2,33 \\
Kaliumsilicat & \\
feucht & 2,21 \\
lufttrocken & 1,99 \\
nach Erhitzen auf $200^{\circ} \mathrm{C}$ & 1,77 \\
\hline
\end{tabular}

* Je nach experimentellen Bedingungen [7].

Das Pulverdiagramm ändert sich dementsprechend mit den äußeren Bedingungen.

Nach Desorption von Zwischenschichtwasser bei etwa $100{ }^{\circ} \mathrm{C}$ oder isothermer Entwässerung bei $\mathrm{p} / \mathrm{p}_{0}<0,4$ beträgt der Schichtabstand etwa $1,80 \mathrm{~nm}$, und die d-Werte des Pulverdiagramms stimmen weitgehend mit den Angaben von Heydemann für Silica-X überein (Tab. II). Die Unterschiede in den Intensitäten hängen mit den Herstellungsbedingungen von Silica-X zusammen. Primär bildet sich das Kaliumsalz, das auch unter stark variierenden Bedingungen und Konzentrationsverhältnissen bevorzugt entsteht. Da die Kaliumionen sehr leicht gegen Protonen ausgetauscht werden und bereits beim Trocknen an der Luft Wasser aus den Schichtzwischenräumen desorbiert wird, entstehen je nach der Art der Weiterbehandlung Präparate mit unterschiedlichem Kaliumionengehalt und etwas unterschiedlichen Pulverdiagrammen. Die Unsicherheit bei der Identifizierung von Silica-X geht auf diese Veränderungen zurück. Die bei der Wiederholung der Heydemannschen Versuche entstehenden Präparate haben unter der Lösung die für das vollhydratisierte Kaliumsilicat typischen Schichtabstände um 2,2 nm, die sich erst beim Auswaschen und Trocknen auf die in der Literatur $[1,2]$ berichteten Werte erniedrigen.

Silica-X bildet sich auch in natriumhydroxid-, nicht aber in lithiumhydroxidhaltiger Lösung $[1,3]$. Bei Gegenwart von Natriumhydroxid entsteht das entsprechende Natriumsilicat, das sich in gleicher Weise wie das Kaliumsilicat in die Kieselsäure umwandelt. Die Bildungsbedingungen für das Natriumsilicat sind enger; zudem bilden sich noch andere Natriumsilicate. Kalium-, Natriumsilicat und die Kieselsäure können durch Kationenaustausch reversibel ineinander umgewandelt werden. Das Natriumsilicat steht in Beziehung zu Kenyait, unterscheidet 
Tab. II. Pulverdiagramme von Silica-X und der Kieselsäure $\mathrm{H}_{2} \mathrm{Si}_{8} \mathrm{O}_{17} \cdot x \mathrm{H}_{2} \mathrm{O}$.

\begin{tabular}{|c|c|c|c|c|c|c|c|c|c|}
\hline \multirow{2}{*}{\multicolumn{2}{|c|}{$\begin{array}{l}\text { Silica-X } \\
\text { (Heydemann, 1964) }\end{array}$}} & \multicolumn{6}{|c|}{ Kristalline Kieselsäure } & \multirow{2}{*}{\multicolumn{2}{|c|}{$\begin{array}{l}\text { bei Zimmertemperatur } \\
\text { lufttrocken }\end{array}$}} \\
\hline & & \multicolumn{2}{|c|}{ über $\mathrm{P}_{4} \mathrm{O}_{10}$} & \multicolumn{2}{|c|}{$\begin{array}{l}\text { bei } 32 \% \\
\text { Feuchtigkeit }\end{array}$} & \multicolumn{2}{|c|}{$\begin{array}{l}\text { nach Erhitzen } \\
\text { auf } 200^{\circ} \mathrm{C}, 24 \mathrm{~h}\end{array}$} & & \\
\hline $\mathrm{I} / \mathrm{I}_{0}$ & $\mathrm{~d}[\mathrm{pm}]$ & $\mathrm{I} / \mathrm{I}_{0}$ & $\mathrm{~d}[\mathrm{pm}]$ & $\mathrm{I} / \mathrm{I}_{0}$ & $\mathrm{~d}[\mathrm{pm}]$ & $\mathrm{I} / \mathrm{I}_{0}$ & $\mathrm{~d}[\mathrm{pm}]$ & $\mathrm{I} / \mathrm{I}_{\mathbf{0}}$ & $\mathrm{d}[\mathrm{pm}]$ \\
\hline 20 & 1800 & 100 & 1803 & 100 & 1785 & 100 & 1776 & 100 & 1900 \\
\hline 10 & 893 & 60 & 884 & 70 & 889 & 70 & 888 & 50 & 930 \\
\hline 12 & 732 & 40 & 728 & 20 & 728 & 40 & 728 & 40 & 728 \\
\hline 16 & 630 & 30 & 663 & 10 & 668 & $<10$ & $668 *$ & & \\
\hline & & 20 & 582 & 10 & 587 & 30 & $585^{*}$ & & \\
\hline 12 & 511 & & & & & & & & \\
\hline 6 & 437 & 30 & 439 & 20 & 444 & 30 & $444^{*}$ & 20 & $449 *$ \\
\hline 6 & 364 & 10 & 366 & & & 30 & 366 & 30 & 364 \\
\hline 100 & 342 & 70 & 338 & 70 & $339 *$ & 70 & $341 * *$ & 70 & 348 \\
\hline & & 20 & 315 & & & & & 60 & $329 *$ \\
\hline 10 & 185 & 50 & 184 & 60 & 184 & 50 & 183 & 50 & 183 \\
\hline & & 15 & 179 & & & & & 25 & 179 \\
\hline
\end{tabular}

* Verbreitert; ** breite Bande.

sich von ihm aber durch die erhöhte Reaktionsfähigkeit. Darüber wird ausführlich berichtet werden.
Neben Silica-X können weitere neue Siliciumdioxidmodifikationen aus kristallinen Kieselsäuren [6] erhalten werden [7].
[1] A. Heydemann, Beitr. Mineral. Petrogr. 10, 242 (1964).

[2] R. Greenwood, Am. Min. 52, 1662 (1967).

[3] P. Bettermann und F. Liebau, Contrib. Mineral. Petrol. 53, 25 (1976).

[4] G. Lagaly und R. Matouschek, Deutsche Patentanmeldung P $2742912.5,1977$.
[5] G. Lagaly, Adv. Colloid Interface Sci. 11, 105 (1979).

[6] G. Lagaly, K. Beneke und A. Weiss, Z. Naturforsch. 28b, 234 (1973) und Am. Min. 60, 642 (1975).

[7] G. Lagaly, K. Beneke und H. Kammermeier, Z. Naturforsch. 34b (1979). 